\title{
Heavy Quarkonium Production in the Regge Limit of QCD
}

\author{
B.A. Kniehl ${ }^{1}$, V.A. Saleev ${ }^{2}$ and D.V. Vasin ${ }^{2}$ \\ 1 - II Institute of Theoretical Physics, University of Hamburg, \\ Luruper Chaussee 149, 22761 Hamburg - Germany \\ 2 - Department of Physics, Samara State University, \\ Samara, ak. Pavlova 1, 443011 Samara - Russia
}

\begin{abstract}
The main results of our recent articles [2, 3] are presented in this report. We study charmonium and bottomonium production at the Tevatron Collider in the framework of the Quasi-Multi-Regge-Kinematic approach and the factorization formalism of nonrelativistic QCD at leading order in the strong-coupling constant $\alpha_{s}$ and the relative velocity $v$ of the heavy quarks inside quarkonium.
\end{abstract}

\section{Introduction}

Heavy quarkonium production at high energies has provided a useful laboratory for testing the high-energy limit of quantum chromodynamics (QCD) as well as the interplay of perturbative and nonperturbative phenomena in QCD. The factorization formalism of nonrelativistic QCD (NRQCD) [4] is a theoretical framework for the description of heavy-quarkonium production and decay. The factorization hypothesis of NRQCD assumes the separation of the effects of long and short distances in heavy-quarkonium production. NRQCD is organized as a perturbative expansion in two small parameters, the strong-coupling constant $\alpha_{s}$ and the relative velocity $v$ of the heavy quarks.

The phenomenology of strong interactions at high energies exhibits a dominant role of gluon interactions in quarkonium production. In the conventional parton model [5], the initial-state gluon dynamics is under the control of the Dokshitzer-Gribov-LipatovAltarelli-Parisi (DGLAP) evolution equations [6]. In this approach, it is assumed that $S>\mu^{2} \gg \Lambda_{\mathrm{QCD}}^{2}$, where $\sqrt{S}$ is the invariant collision energy, $\mu$ is the typical energy scale of the hard interaction, and $\Lambda_{\mathrm{QCD}}$ is the asymptotic scale parameter. In this way, the DGLAP evolution equation takes into account only one large logarithm, namely $\ln \left(\mu / \Lambda_{Q C D}\right)$. In fact, the collinear approximation is used, and the transverse momenta of the initial gluons are neglected.

In the high-energy limit, the contribution from the partonic subprocesses involving $t$ channel gluon exchanges to the total cross section becomes dominant. The summation of the large logarithms $\ln (\sqrt{S} / \mu)$ in the evolution equation can then be more important than the one of the $\ln \left(\mu / \Lambda_{Q C D}\right)$ terms. In this case, the non-collinear gluon dynamics is described by the Balitsky-Fadin-Kuraev-Lipatov (BFKL) evolution equation [7]. In the region under consideration, the transverse momenta $\left(k_{T}\right)$ of the incoming gluons and their off-shell properties can no longer be neglected, and we deal with reggeized $t$-channel gluons. The theoretical framework for this kind of high-energy phenomenology is the Quasi-MultiRegge-Kinematic (QMRK) approach [8], which can be based on effective quantum field theory implemented with the non-abelian gauge-invariant action, as was suggested a few years ago [9]. 


\section{Charmonium production at the Tevatron}

During the last decade, the CDF Collaboration at the Tevatron $[10,11]$ collected data on charmonium production at energies $\sqrt{S}=1.8 \mathrm{TeV}$ (run I) and $\sqrt{S}=1.96 \mathrm{TeV}$ (run II) in the central region of pseudorapidity $|\eta|<0.6$. The data cover a large interval in transverse momentum, namely $5<$ $\left|\mathbf{p}_{T}\right|<20 \mathrm{GeV}$ (run I) and $\left|\mathbf{p}_{T}\right|<20 \mathrm{GeV}$ (run II). The data sample of run I [10] includes transverse-momentum distributions of $J / \psi$ mesons that were produced directly in the hard interaction, via radiative decays of $\chi_{c J}$ mesons, via decays of $\psi^{\prime}$ mesons, and via decays of $b$ hadrons. That of run II [11] includes transverse-momentum distributions of prompt $J / \psi$ mesons, so far without separation into direct, $\chi_{c J}$-decay, and $\psi^{\prime}$-decay contributions, and $J / \psi$ mesons from $b$-hadron decays.

In contrast to previous analyses in the collinear parton model [12] or the $k_{T^{-}}$ factorization approach [13], we perform a joint fit to the run-I and run-II CDF data $[10,11]$ to obtain the color-octet nonperturbative matrix elements (NMEs) for $J / \psi$, $\chi_{c J}$, and $\psi^{\prime}$ mesons. Our calculations are based on exact analytical expressions for the relevant squared amplitudes, which were previously unknown in the literature. Our fits include five experimental data sets, which come as $p_{T}$ distributions of $J / \psi$

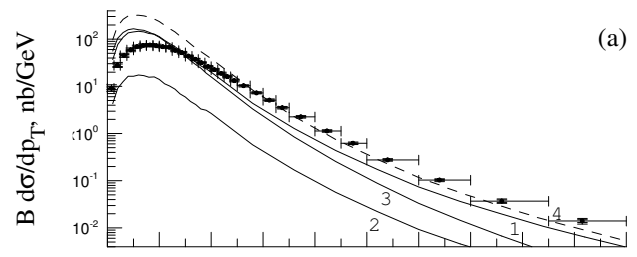

(a)
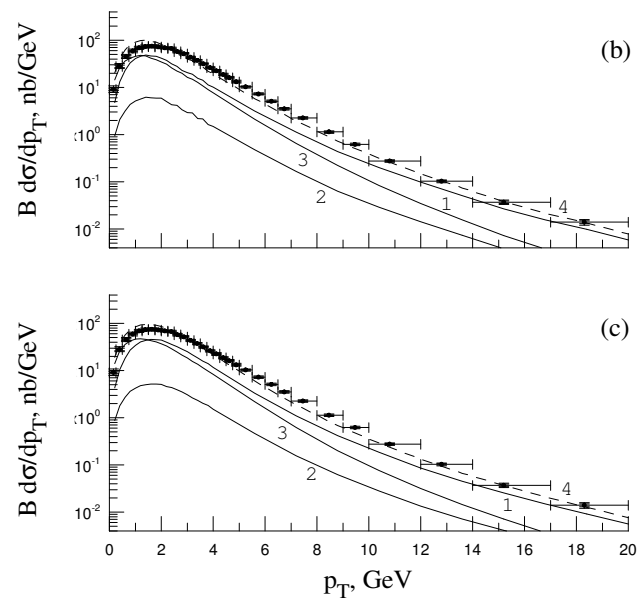

Figure 1: Prompt $J / \psi$ production at the Tevatron with $\sqrt{S}=1.96 \mathrm{TeV}[11]$. The total theoretical results (4) obtained with the (a) JB, (b) JS, or (c) KMR unintegrated gluon distributions are broken down to the contributions from (1) direct production, (2) $\psi^{\prime}$ decays, and (3) $\chi_{c J}$ decays. mesons from direct production, prompt production, $\chi_{c J}$ decays, and $\psi^{\prime}$ decays in run I, and from prompt production in run II.

In Table I of Ref. [2], we present out fit results for the relevant color-octet NMEs for three different choices of unintegrated gluon distribution function, namely JB [14], JS [15], and KMR [16]. The first one, JB, is strongly disfavoured by the charmonium data in the small $p_{T}$ region, as it is shown in Fig. 1(a).

Considering the color-octet NMEs relevant for the $J / \psi, \psi^{\prime}$ and $\chi_{c J}$ production mechanisms, we can formulate the following heuristic rule for favoured transitions from color-octet to color-singlet states: $\Delta L \simeq 0$ and $\Delta S \simeq 0$; i.e., these transitions are doubly chromoelectric and preserve the orbital angular momentum and the spin of the heavy-quark bound state. 


\section{Bottomonium production at the Tevatron}

The CDF Collaboration measured the $p_{T}$ distributions of $\Upsilon(1 S), \Upsilon(2 S)$, and $\Upsilon(3 S)$ mesons in the central region of rapidity $(y)$, $|y|<0.4$, at $\sqrt{S}=1.8 \mathrm{TeV}$ (run I) $[17]$ and that of the $\Upsilon(1 S)$ meson in the rapidity regions $|y|<0.6,0.6<|y|<1.2$, and $1.2<|y|<1.8$ at $\sqrt{S}=1.96 \mathrm{TeV}$ (run II) [18]. In both cases, the $S$-wave bottomonia were produced promptly, i.e., directly or through non-forbidden decays of higher-lying $S$ - and $P$-wave bottomonium states, including cascade transitions such as $\Upsilon(3 S) \rightarrow \chi_{b 1}(2 P) \rightarrow \Upsilon(1 S)$.

In contrast to previous analysis in the collinear parton model [19], we perform a joint fit to the CDF data from run I [17] and run II [18] for all $p_{T}$ values, including the small- $p_{T}$ region. Comparing the color-singlet and color-octet contributions, we observe that the latter is dominant in the $\Upsilon(3 S)$ case and in the $\Upsilon(2 S)$ case for $p_{T} \gtrsim 13 \mathrm{GeV}$, while it is of minor importance in the $\Upsilon(1 S)$ case in the whole $p_{T}$ range considered. The fits based on the KMR, JB, and JS gluons turned out to be excellent, fair, and poor, respectively. They yielded small to vanishing values for the color-octet NMEs, see Table II of Ref. [3], especially when the estimated feed-down contributions from the as-yet unobserved $\chi_{b J}(3 P)$ states were included.

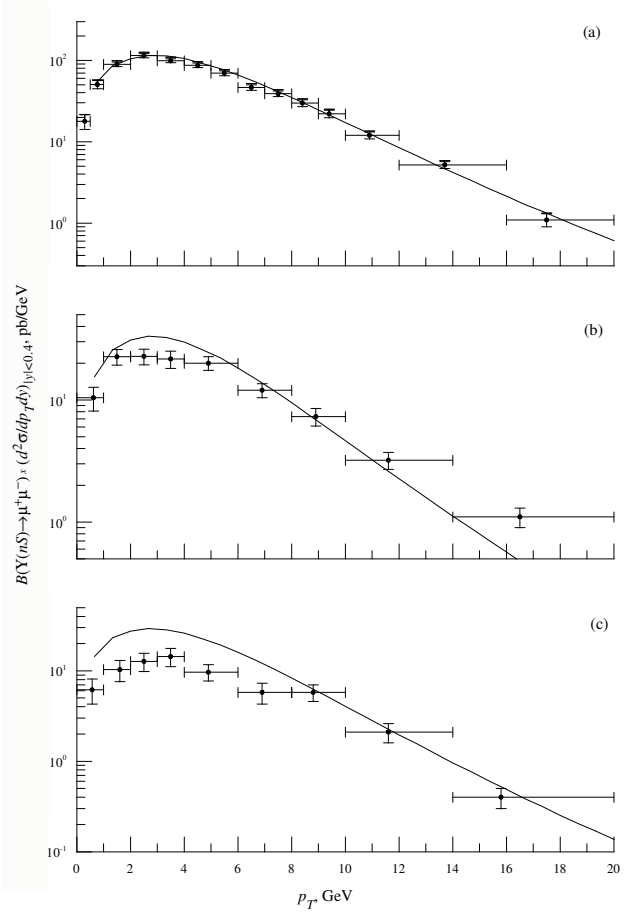

Figure 2: Prompt production of (a) $\Upsilon(1 S)$, (b) $\Upsilon(2 S)$, and (c) $\Upsilon(3 S)$ mesons at the Tevatron with $\sqrt{S}=1.8 \mathrm{TeV}$ [17]. The theoretical results are obtained with the KMR unintegrated gluon distribution and correspond to the color-singlet contributions, also including the estimated contribution due to the $\chi_{b J}(3 P)$ meson.

\section{Conclusions}

Working at LO in the QMRK approach to NRQCD, we analytically evaluated the squared amplitudes of prompt quarkonium production by reggeized gluons. We extracted the relevant color-octet NMEs, $\left\langle\mathcal{O}^{\mathcal{H}}\left[{ }^{3} S_{1}^{(8)}\right]\right\rangle$, $\left\langle\mathcal{O}^{\mathcal{H}}\left[{ }^{1} S_{0}^{(8)}\right]\right\rangle$, and $\left\langle\mathcal{O}^{\mathcal{H}}\left[{ }^{3} P_{0}^{(8)}\right]\right\rangle$ for $\mathcal{H}=\psi(1 S, 2 S), \chi_{c J}(1 P), \Upsilon(1 S, 2 S, 3 S)$, and $\chi_{b J}(1 P, 2 P)$ through fits to $p_{T}$ distributions measured by the CDF Collaboration in $p \bar{p}$ collisions at the Tevatron with $\sqrt{S}=1.8 \mathrm{TeV}[10,17]$ and $1.96 \mathrm{TeV}[11,18]$ using various unintegrated gluon distribution functions. The present analysis suggest that the color-octet NMEs of bottomonium are more strongly suppressed than those of charmonium as expected from the velocity scaling rules of NRQCD. We illustrated that the QMRK approach [9] provides a useful laboratory to describe the phenomenology of high-energy processes in the Regge limit of QCD. 


\section{Acknowledgements}

V.A.S. thanks the DIS07 Local Organizing Committee for the kind hospitality during the conference and for the financial support. The work of B.A.K. was supported in part by the German Federal Ministry for Education and Research BMBF through Grant No. 05 HT6GUA and by the German Research Foundation DFG through Grant No. KN 365/61 .

\section{References}

[1] Slides: http: //indico. cern. ch/contributionDisplay py? contribId=79\&sessionId=13\&conf Id=9499

[2] B. A. Kniehl, D. V. Vasin, and V. A. Saleev, Phys. Rev. D 73, 074022 (2006).

[3] B. A. Kniehl, V. A. Saleev, and D. V. Vasin, Phys. Rev. D 74, 014024 (2006).

[4] G. T. Bodwin, E. Braaten, and G. P. Lepage, Phys. Rev. D 51, 1125 (1995); 55, 5853(E) (1997).

[5] CTEQ Collaboration, R. Brock et al., Rev. Mod. Phys. 67, 157 (1995).

[6] V. N. Gribov and L. N. Lipatov, Sov. J. Nucl. Phys. 15, 438 (1972) [Yad. Fiz. 15, 781 (1972)]; Yu. L. Dokshitzer, Sov. Phys. JETP 46, 641 (1977) [Zh. Eksp. Teor. Fiz. 73, 1216 (1977)]; G. Altarelli and G. Parisi, Nucl. Phys. B 126, 298 (1977).

[7] E. A. Kuraev, L. N. Lipatov, and V. S. Fadin, Sov. Phys. JETP 44, 443 (1976) [Zh. Eksp. Teor. Fiz. 71, 840 (1976)]; I. I. Balitsky and L. N. Lipatov, Sov. J. Nucl. Phys. 28, 822 (1978) [Yad. Fiz. 28, 1597 $(1978)]$.

[8] V. S. Fadin and L. N. Lipatov, Nucl. Phys. B477, 767 (1996).

[9] L. N. Lipatov, Nucl. Phys. B 452, 369 (1995); E. N. Antonov, L. N. Lipatov, E. A. Kuraev, and I. O. Cherednikov, Nucl. Phys. B 721, 111 (2005).

[10] CDF Collaboration, F. Abe et al., Phys. Rev. Lett. 79, 572 (1997); 79, 578 (1997); CDF Collaboration, T. Affolder et al., Phys. Rev. Lett. 85, 2886 (2000).

[11] CDF Collaboration, D. Acosta et al., Phys. Rev. D 71, 032001 (2005).

[12] G. T. Bodwin, E. Braaten, and J. Lee, Phys. Rev. D 72, 014004 (2005).

[13] Ph. Hägler, R. Kirschner, A. Schäfer, L. Szymanowski, and O. V. Teryaev, Phys. Rev. D 62, 071502 (2000); Phys. Rev. Lett. 86, 1446 (2001); F. Yuan and K.-T. Chao, Phys. Rev. D 63, 034006 (2001); Phys. Rev. Lett. 87, 022002 (2001).

[14] J. Blümlein, Report No. DESY 95-121 (1995).

[15] H. Jung and G. P. Salam, Eur. Phys. J. C 19, 351 (2001).

[16] M. A. Kimber, A. D. Martin, and M. G. Ryskin, Phys. Rev. D 63, 114027 (2001).

[17] CDF Collaboration, F. Abe et al., Phys. Rev. Lett. 75, 4358 (1995); CDF Collaboration, D. Acosta et al., ibid. 88, 161802 (2002).

[18] CDF Collaboration, V. M. Abazov et al., Phys. Rev. Lett. 94, 232001 (2005).

[19] E. Braaten, S. Fleming, and A. K. Leibovich, Phys. Rev. D 63, 094006 (2001). 